\title{
MACRO STRESS MAPPING ON THIN FILM BUCKLING
}

\author{
P. Goudeau ${ }^{1}$, P. Villain ${ }^{1}$, P.-O. Renault ${ }^{1}$, N. Tamura ${ }^{2}$, R.S. Celestre ${ }^{2}$ \\ and $\mathrm{H}$. Padmore ${ }^{2}$
}

${ }^{1}$ Laboratoire de Métallurgie Physique, UMR 6630 CNRS, Université de Poitiers, SP2MI, Téléport2, Bd M. et P. Curie, BP 30179, F-86962 Futuroscope Chasseneuil cedex

${ }^{2}$ Advanced Light Source, Lawrence Berkeley National Laboratory, 1 Cyclotron Road, MS 2-400, Berkeley, CA, 94270, USA

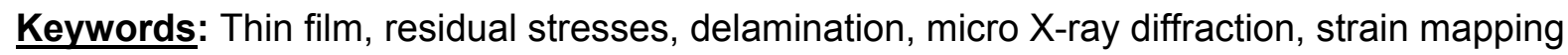

\begin{abstract}
Thin films deposited by Physical Vapour Deposition techniques on substrates generally exhibit large residual stresses which may be responsible of thin film buckling in the case of compressive stresses. Since the 80's, a lot of theoretical work has been done to develop mechanical models but only a few experimental work has been done on this subject to support these theoretical approaches and nothing concerning local stress measurement mainly because of the small dimension of the buckling (few $10^{\text {th }} \mu \mathrm{m}$ ). This paper deals with the application of micro beam Xray diffraction available on synchrotron radiation sources for stress mapping analysis of gold thin film buckling.
\end{abstract}

\section{Introduction}

The development of thin films for industrial applications is constantly increasing because deposition techniques provide new class of materials which are impossible to obtain with classical elaboration processes. The structure which develops during thin film growth is tightly controlled by the deposition process. Thin films deposited by sputtering technique on non epitaxial substrates are often nanocrystallines which confers to the film very interesting properties. Depending on deposited atom energy, thin films adherent to bulk substrates are generally in a tensile or compressive residual stress state which may affect their physical properties and their life time.

In the case of thin films deposited at room temperature by direct ion beam sputtering, biaxial compressive residual stresses in the sample plane and interstitial defects in the unit cell are generally observed. The stress magnitude is often very high (larger than the elastic limit of the same material in the bulk state) and thus spontaneous delamination phenomenon such as 1D - wrinkling (see figure 1) or 2D - buckling (figure 2) may appear for a critical film thickness (relaxation of the stored elastic energy) when extracting the sample out side the deposition chamber [1-4].

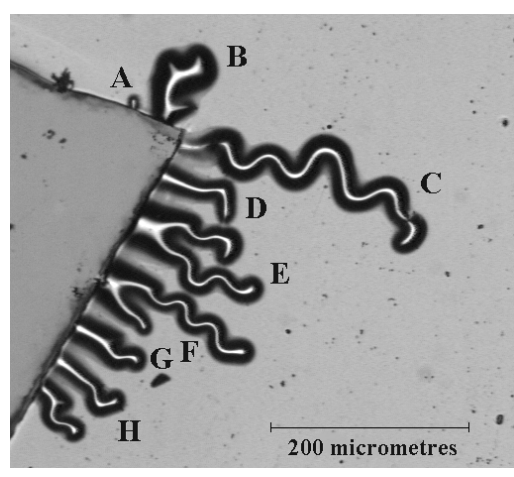

(a)

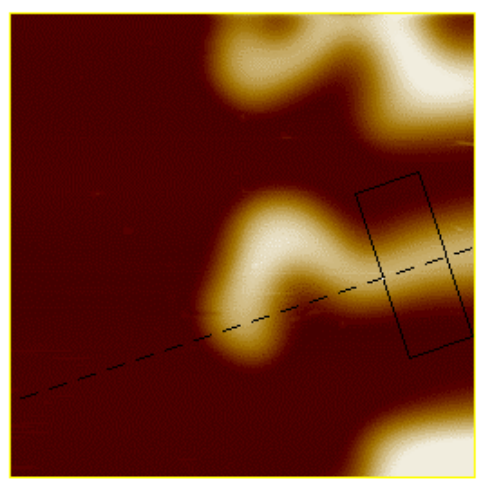

(b)

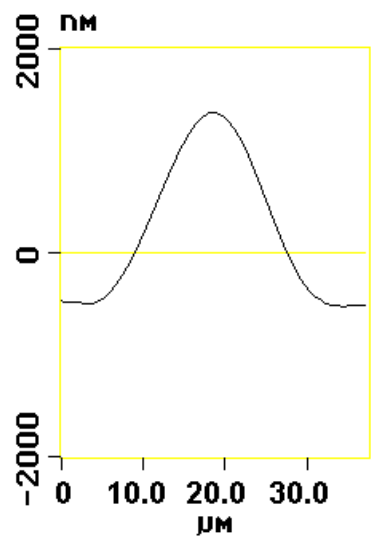

(c) 
Figure 1. Wrinkles observed by optical microscopy on a 304L steel film $500 \mathrm{~nm}$ thick deposited on 630 um Silicon wafer (a) close to a step realised during the deposition process for film thickness measurement and AFM images (b) of buckling noted D on (a) and corresponding cross section, (c) perpendicular to the propagation direction.

Although a lot of theoretical works have been done [5] to develop mechanical models and calculations (elasticity of thin plates, fracture mechanic, finite element and analytical calculations) with the aim to get a better understanding of driven mechanisms giving rise to this phenomenon, only a few experimental work [1-2] has been done on this subject to support these theoretical results and nothing concerning local stress/strain measurement mainly because of the small dimension of the buckling (few tenth $\mu \mathrm{m}$ ).

In this paper we use micro beam X-ray diffraction ( $\mu$-XRD) available on synchrotron radiation sources as a local probe (spatial) for stress/strain analysis of gold thin film buckling [6, 7].

\section{Spontaneous Buckling: Gold films}

$630 \mathrm{~nm}$ thick gold films deposited on silicon (100) substrates covered with native oxide have been chosen for these measurements. The delamination of the thin film is evidenced on figure 2 (a) and an individual buckle is shown on figure 2 (b); its shape corresponds to a portion of a sphere.

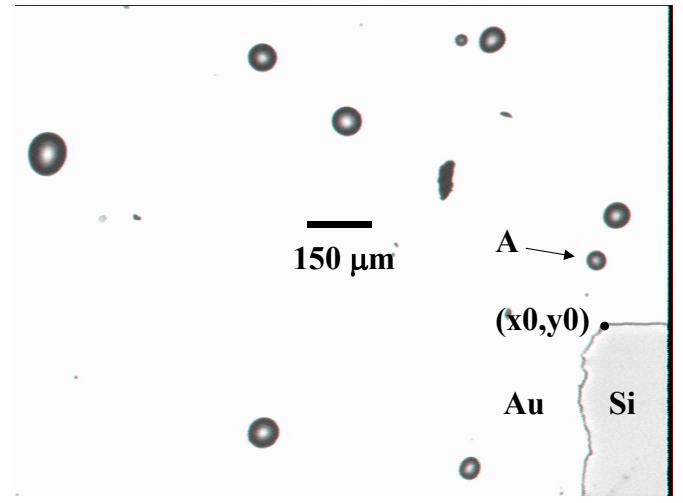

(a)

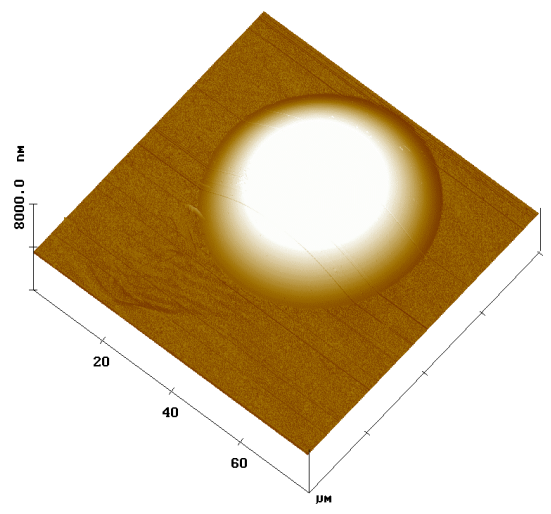

(b)

Figure 2. $630 \mathrm{~nm}$ gold film sputter deposited on Si substrate: (a) optical image of the sample surface and (b) Atomic Force Microscopy image of the buckle noted A on fig. (a); the in plane width is around $40 \mu \mathrm{m}$ and the height of $1.7 \mu \mathrm{m}$.

Wang and Evans [8] have established a simple relation ship between the width L and the height $\delta$ of the 2D buckling from which the residual stresses $\sigma$ may be determined using elastic constants of the bulk gold (Young Modulus E=80.2 GPa and Poisson's ratio v=0.42):

$$
\mathrm{L}=\mathrm{A} \delta
$$

Where $\mathrm{A}=\left((4 / \sigma)^{*} \mathrm{E}_{\mathrm{f}} / 1-v \mathrm{f}^{2}\right)^{1 / 2}$ and $\left.\delta^{\prime}=(\delta / 1.25)^{2}+\left(1.22^{*} \mathrm{~h}^{2}\right)\right) 1 / 2 ; \mathrm{h}$ is the film thickness.

The data obtained by Atomic Force Microscopy on the different buckling sizes shown on figure 2 are plotted on figure 3. The stress value deduced from the slope of the linear fit is around -290 $\mathrm{MPa}(0.4 \%$ for strains $)$.

\section{Macro stress analysis by X-ray diffraction}


Among the most widely used method for determining the stress level in thin films, X-ray diffraction (XRD) is phase selective and the unique non destructive technique which allows to determine both the mechanical and microstructural state of the diffracting phases. Indeed, the distance between atomic planes is used as an internal strain gage. For polycrystalline samples, the measurement of the diffraction peak position shift using $\sin ^{2} \psi$ method allows to extract the stress tensor and the stress free lattice parameter [9]. However, x-ray diffraction is difficult to use in low dimensional systems because the diffracted intensities are weak due to the reduced thicknesses and nanocrystalline character (grain size) of such materials. These problems may be solved using intense $x$-ray sources such as synchrotron radiation (S.R.). In addition to the high flux characteristic of S.R. facilities, the wide wavelength spectra and the optics (micro beam) which are available on beam lines $\left(3^{\text {rd }}\right.$ generation SR only) allow to perform specific XRD experiments which are not possible with classical X-ray sources in laboratories.

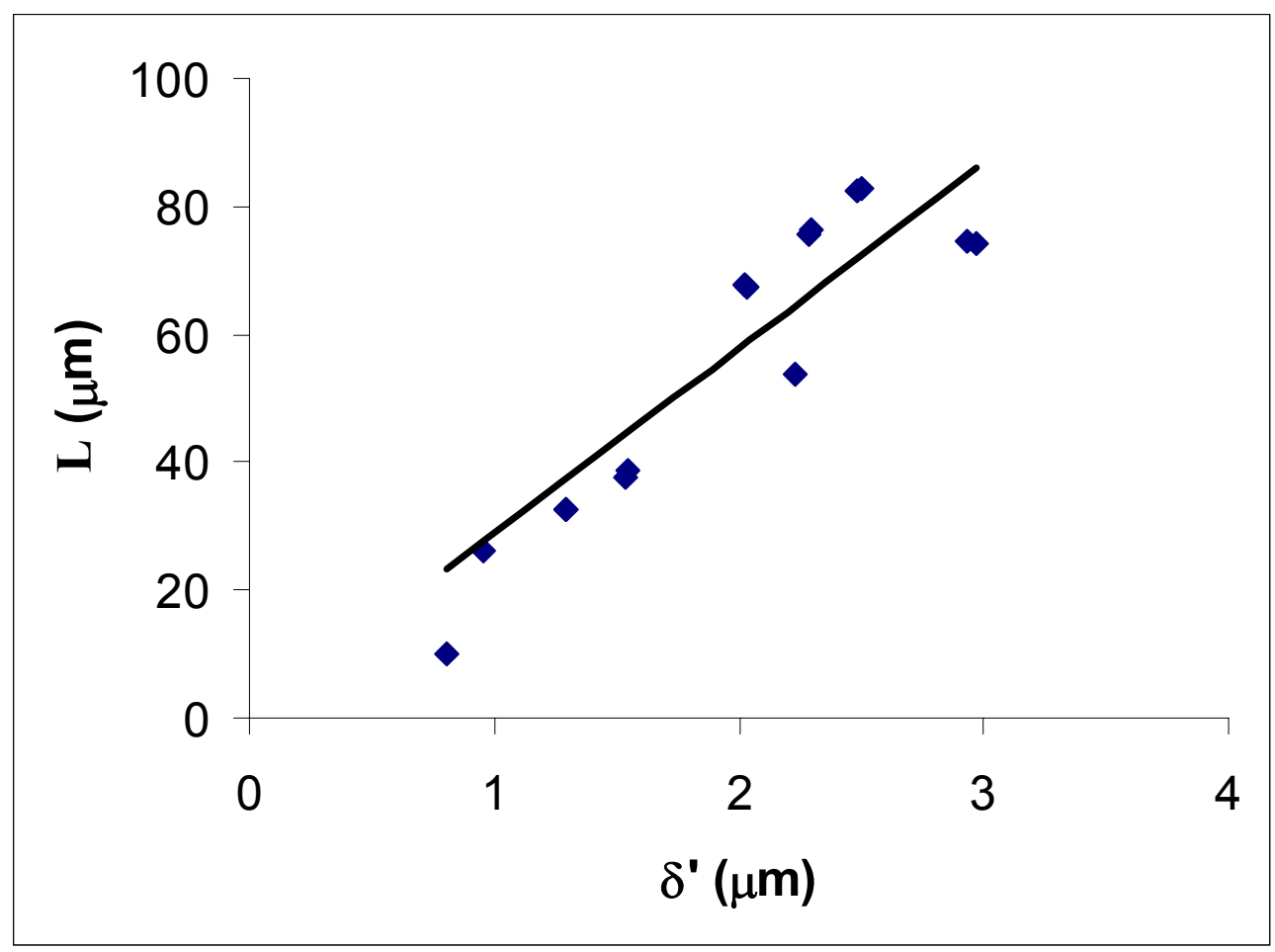

Figure 3. Variation of the buckle width $\mathrm{L}$ as a function its corrected height $\delta$ '. The straight line represents the linear fit.

The 7.3.3.1 Microdiffraction beam line [10] at Advanced Light Source of the Lawrence Berkeley National Laboratory [11] provides a reduced spot size less than $1 \mu^{2}$ (Kirkpatrick Baez mirrors) with high flux for white or monochromatic radiation (4-crystals channel cut monochromator: 6-14 KeV; see figure 4 and 5). These performances are unique and perfectly adapted to our purpose.

For comparison, the Micro-Fluorescence-Imaging-Diffraction group (ID22) at ESRF has developed Bragg-Fresnel lens [12] which allow to get reduced spot size greater than $1 \times 5 \mathrm{~m}^{2}$ with high flux for an higher energy range (up to $30 \mathrm{keV}$ ). On the same facility, ID13 beam line achieves $2 \mu \mathrm{m}$ diameter spot size with the use of capillaries [13]. At the National Synchrotron Light Source of Brookhaven National Laboratory in New-York (USA), micro beam X-ray diffraction is used to get a topography of the sample surface in order to determine strains at a scale less than a $\mu \mathrm{m}$ [14]. We can find also specific micro beam lines at the Photon Factory - Japan [15]. The proceeding of the $7^{\text {th }}$ International Conference on Synchrotron Radiation Instrumentation (Berlin, Germany, 
August 2000) gives an overview of the different facilities all over the world where $\mu$-XRD is available [10, 13-16].

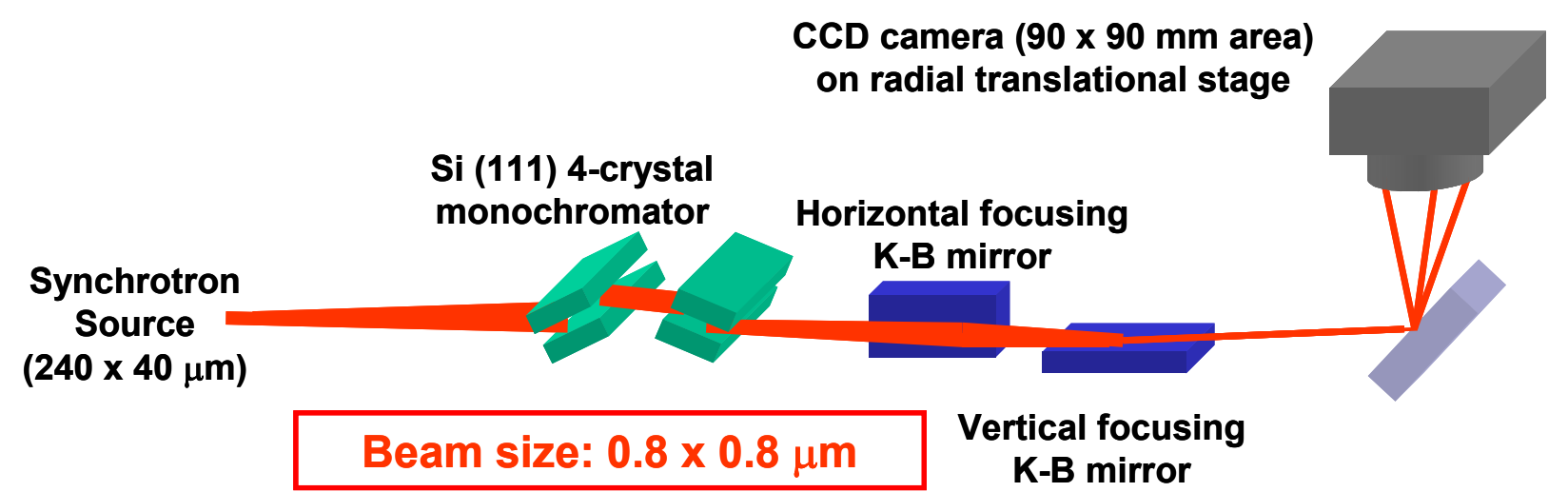

Figure 4. Schematic drawing of $\mu$-XRD beam line 7.3.3.1 at the A. L. S. Berkeley - USA.
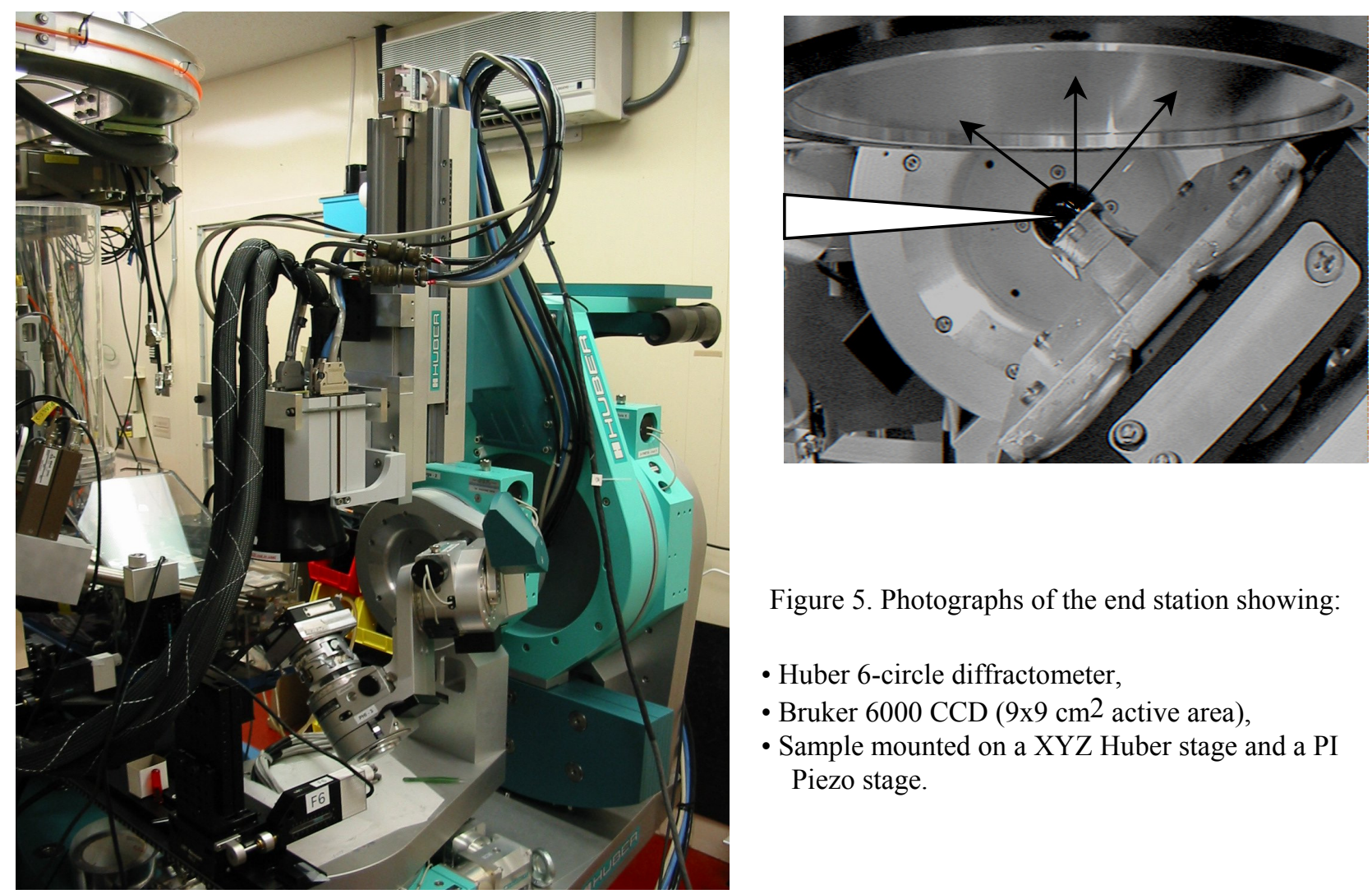

Figure 5. Photographs of the end station showing:

- Huber 6-circle diffractometer,

- Bruker $6000 \mathrm{CCD}\left(9 \times 9 \mathrm{~cm}^{2}\right.$ active area),

- Sample mounted on a XYZ Huber stage and a PI Piezo stage.

A lot of XRD experiment has been done to investigate in bulk materials the mechanical properties of each individual grains with micro beam [16-18] or white beam [19] in a high energy range.

In our gold samples, the grain size $(\leq 10 \mathrm{~nm})$ is smaller than the X-ray beam one and macrostrains are of about $0.4 \%$. Furthermore, rocking curve measurements have evidenced strong $<111>$ fiber texture in the films. The diffraction pattern recorded with a 2D CCD detector is composed of different rings which allow to extract (using specific software - to be developed) the in-plane stress without any tilt of the samples (the $\psi$ description is contained in the $2 \mathrm{D}$ pattern and the angular resolution is enough to appreciate such large macro-strains). Accurate spatial spot localization on the sample surface is achieved from markers delimitating the region of interest. A precise 
goniometer allows XRD measurements in reflection mode and an $\mathrm{x}-\mathrm{y}$ translation stage is used for scanning the sample surface.

\section{Results and concluding remarks}

Preliminary micro beam X-ray diffraction experiments have been successfully done in June 2001 on the buckle noted A on figure 1. The position of the buckle $(\mathrm{x}, \mathrm{y})$ is determined from the step corner coordinates $(\mathrm{x} 0, \mathrm{y} 0)$ which have been measured by X-ray fluorescence (white beam). The absolute accuracy on the position is better than $10 \mu \mathrm{m}$. Let us notice that this step on the silicon wafer has been realized during the film deposition.

The calibration of the 2D diffraction diagram is done from the diffraction of the single crystal silicon substrate using white X-ray beam. The diffraction pattern obtained in an adherent (flat) region of the gold film is shown in figure 6 . The $2 \Theta$ angular position of the (311) diffraction ring is around $120^{\circ}$ for the considered photon energy and sample configuration; the $\mathrm{X}$ angular range is 0 $\pm 55^{\circ}$. Because of the $<111>$ texture of the film, we observe maximum intensities on each two rings for particular pole directions X. During the scan of the buckle A with a $10 \mu \mathrm{m}$ step, the position of the two maximum intensities ( $\mathrm{X}+$ and $\mathrm{X}$-) moves symmetrically (fig. 6b) or asymmetrically (fig. 6c) along the ring because of the rotation of the normal to the film surface around one axis $\mathrm{x}$ (fig. $6 \mathrm{~b}$ ) or two axis, $x$ and $y$ (fig. $6 \mathrm{c}$ ). Thus, from the diffraction pattern, one may deduce the position of the beam on the buckle and the corresponding strains. A comparison between the diffraction diagrams obtained in flat regions and at the top of the buckle indicates a stress relaxation.
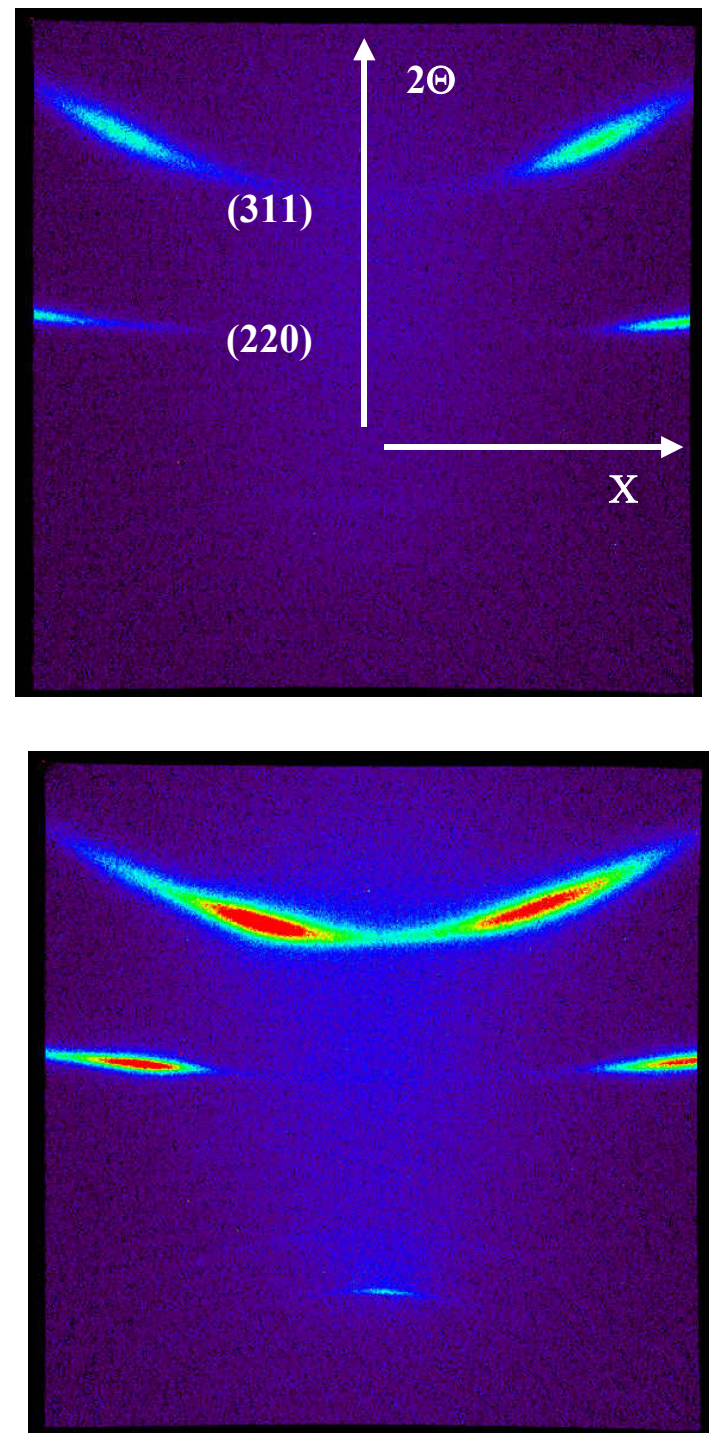

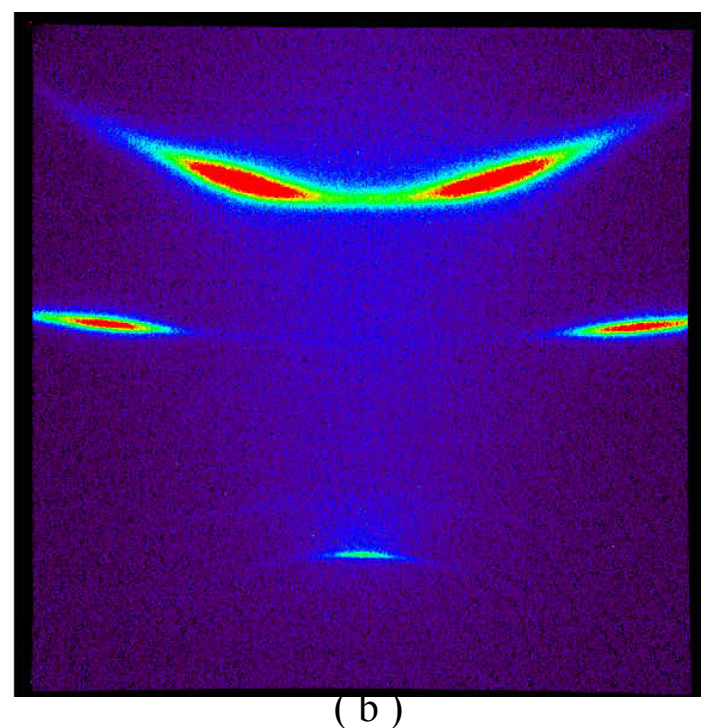

Figure 6: 2D X-ray diffraction diagrams obtained on an adherent gold film region (a) and on two different parts of the buckling (b), (c) with an X-ray energy of 5.7 $\mathrm{keV}$, a spot size on the sample of $3 \times 3 \mathrm{~m}^{2}$ and a recording time of $300 \mathrm{sec}$. 
(c)

These first measurements show the feasibility of such an experiment. The evolution of the 2D diffraction diagram during $\mathrm{x}-\mathrm{y}$ scan is directly related to the buckling shape; the position of the beam on the buckling is thus precisely known. A relaxation of the compressive stress is observed when comparing the diffraction diagram measured on adherent region and the one corresponding to the top of the blister. A complete interpretation of the data is still under progress because specific tools and methodologies have to be applied for complete diffraction pattern analysis. Accuracy and sensibility are the two main points to be considered for strain variation during the scan.

This study will be completed by new $\mu$-XRD experiments in transmission mode and at higher photon energies (proposal submitted to ESRF). Furthermore, Finite Element Calculations have been engaged for determining stress map in such spherical gold buckles which will be confronted to $\mu$-XRD results.

\section{Acknowledgements}

The authors would like to thank M. Drouet for gold thin film deposition and C. Coupeau for Atomic Force Microscopy observations.

The Advanced Light Source is supported by the Director, Office of Science, Office of Basic Energy

Sciences, Materials Sciences Division, of the U.S. Department of Energy under Contract No DEAC03-76SF00098 at Lawrence Berkeley National Laboratory.

\section{References}

[1] Talea M., Boubeker B., Cleymand F., Coupeau C., Grilhé J., Goudeau Ph., Materials letters 41 181 (1999).

[2] Coupeau C., Naud J.-F., Cleymand F., Goudeau P., Grilhé J., Thin Solid Films 353194 (1999).

[3] Branger V., Coupeau C., Goudeau P., Boubeker B., Badawi K.F., Grilhé J., J. of Materials Science Letters 19353 (2000).

[4] Boubeker B., Talea M., Goudeau Ph., Coupeau C., Grilhé J., Materials Charac. 4533 (2000).

[5] Gioia G. et Ortiz M., Advances in Applied Mechanics 33119 (1997).

[6] Goudeau P. in PHOTOMECANIQUE 2001 ed. Y. Bertaud et col. (GAMAC, Paris, 2001) p. 87.

[7] Goudeau P., Villain P., Tamura N., Celestre R.S., Padmore H., submitted to J. Phys. IV

[8] Wang J.-S. and Evans A.G., Acta Mater. 46 (14) 4993 (1998)

[9] Branger V., Pelosin V., Goudeau P., Badawi K.F., High Temp. Material Pro. 2 (1998) p. 419

[10] MacDowell A.A., Celestre R.S., Tamura N., Spolenak R., Valek B., Brown W.L., Bravman J.C., Padmore H.A., Batterman B.W. and Patel J.R., N.I.M. in Phys. A 467-468 (2001) p. 936.

[11] http://www-als.lbl.gov

[12] Snigirev A., Rev. Sci. Instrum. 66 (2) 2053 (1995).

[13] Muller M., Burghammer M., Riekel Ch., ., N.I.M. in Phys. A 467-468 (2001) p. 958.

[14] Hanan J.C., Ustundag E., Aydiner C.C., Kaldor S.K., Noyan I.C., T.M.S. Annual Meeting, Symposium "Deformation and Stresses in Small Volume", Seattle (USA), February 2002.

[15] Noma T., Takada K., Miyata H., Iida A., N.I.M. in Phys. A 467-468 (2001) p. 1021.

[16] Castelnau O., Drakopoulos M., Snigirev A., Snigireva I., Schroer C., Chauveau T., Bacroix B., Ungar T., N.I.M. in Phys. A 467-468 (2001) p. 1245.

[17] Biermann H., Grossmann B.V., Mechsner S., Mughrabi H., Ungar T., Snigirev A., Snigireva I., Souvorov A., Kocsis M., Raven C., Scripta Materiala 37 (9) 1309 (1997).

[18] Maire E., Owen A., Buffiere J.-Y., Withers P.J., Acta Mater. 49 (2001) p. 153.

[19] Genzel Ch., Stock C., Wallis B., Reimers W., N.I.M. in Phys. A 467-468 (2001) p. 1253. 